handlung dieser Volksinitiative im Nationalrat hat der Vorsteher des Departementes des Innem das Gesundheitsamt beauftragt, die Arbeiten für das Präventivgesetz zu beschleunigen. Es ist vorgesehen, diese Gesetzesnovelle im Verlaufe der nächsten Legislaturperiode (1979/1983) den Räten zu unterbreiten.

Es soll sich um ein einfaches Rahmengesetz handeln, das den Schutz und die Förderung der Gesundheit durch primär-präventive, also vor allem erzieherische Massnahmen zum Ziele hat. Der Geltungsbereich soll sich nur auf Krankheiten, Gebrechen und Unfälle beschränken, die durch präventive Massnahmen verhütet oder günstig beeinflusst werden können. Es wird also an einen restriktiven Geltungsbereich gedacht. Aufgabe der Kantone wäre es, die Gesundheitserziehung durchzuführen, gefährdete Bevölkerungsgruppen aufzuklären, die Aus- und Weiterbildung von Fachpersonal zu fördern. Die Kantone sollen möglichst regional und interkantonal zusammenarbeiten. Dem Bund käme die Aufgabe zu, die Kantone und geeignete private Organisationen durch Dienstleistungen wie Statistiken, Dokumentation, Information usw. zu unterstützen. Er soll die Tätigkeit der Kantone und der privaten Organisationen fördern und koordinieren. Gegebenenfalls kann er ein konsultatives Koordinationsorgan einsetzen und gesamtschweizerische Vorbeugungsmassnahmen in Zusammenarbeit mit den
Kantonen und privaten Organisationen durchführen. Schliesslich soll der Bund die wissenschaftliche Forschung über gesundheitserzieherische und andere präventive Massnahmen fördern. Wichtig ist vor allem die Möglichkeit, dass bestimmte Aufgaben und Befugnisse privaten Organisationen übertragen werden können.

Unklarheit herrscht noch über das Ausmass allfälliger finanzieller Mehraufwendungen des Bundes und deren mögliche Deckung. Vielleicht müssen neue Finanzierungsquellen erschlossen werden. Wichtig scheint uns, dass das Verursacherprinzip angemessen berücksichtigt wird.

Das Gesundheitsamt würde es begrüssen, wenn sich die hier in Davos versammelte Gruppe von Spezialisten und Politikern mit diesem Projekt etwas näher befassen könnte. Die Bearbeitung der vorgelegten Projekt-Skizze durch die Arbeitsgruppe «Gesetzgebung» wäre den Bundesbehörden für die Erledigung des ihnen übertragenen Auftrags eine grosse Hilfe.

\section{Summary}

Prevention in Swiss legislation

History and present situation of legislation on the cantonal and federal levels are briefly discussed. Special attention is given to the planned federal law on prevention which should provide a frame for various measures aiming at primary prevention of illnesses and accidents.

\title{
Prévention dans la législation fédérale
}

\section{Dr U. Frey}

L'auteur rappelle que la plupart des lois fédérales dans le domaine sanitaire concernent la protection de la santé (LF sur les denrées alimentaires, sur la lutte contre les maladies transmissibles, sur la protection contre les radiations, sur le commerce des toxiques, ainsi que sur l'organisation et la surveillance des examens des professions médicales). Il n'y a pas à proprement parler de loi fédérale sur la santé publique, qui est pour l'essentiel de la compétence des cantons. Plusieurs des lois fédérales ci-dessus mentionnées ont récemment été (ou sont actuellement) l'objet de révisions importantes. Alors même que certains parlent d'une «inflation législative» de la part de la Confédération, il est considéré comme raisonnable de penser à une loi sur la prévention au niveau fédéral, et le Service fédéral de l'hygiène publique (SFHP) a entrepris des travaux préparatoires en vue d'élaborer un projet traitant des mesures de prévention vis-à-vis de maladies largement répandues ou malignes.

En 1972 déjà, le Conseiller fédéral Tschudi avait chargé le SFHP de préparer une loi sur les toxicomanies. Un groupe de travail ad hoc êtait arrivé à la conclusion que l'art. 69 de la Constitution fédérale donnait la base légale nécessaire. Cette base, de l'avis des juristes consultés, permet aussi d'envisager la pro- mulgation d'une Loi sur la prévention. Les travaux du groupe ad hoc montrèrent qu'une loi sur les toxicomanies serait probablement trop limitée et qu'il fallait plutôt penser à une loi-cadre qui embrasse tout le champ de la prévention (notion des multiples types de facteurs de risque, en plus des toxicomanies et abus «classiques»).

Les réflexions du groupe furent soumises aux cantons et aux autres cercles intéressés dans le cadre d'une consultation. Bien que les opinions ne soient pas unanimes, la plupart des cantons acceptèrent l'idée d'une loi-cadre qui donnerait à la Confédération des fonctions de coordination dans le domaine de la prévention primaire (y compris éducation pour la santé), de l'information et de la documentation. Suite à l'initiative récente dite «des Bons Templiers», le Chef du Département de l'Intérieur a demandé au SFHP d'accélérer les travaux préparatoires d'une telle loi, qui pourrait être soumise aux Chambres dans le courant de la législature 1979-1983. Il convient d'insister sur le fait qu'il s'agirait d'une loi-cadre, dont le domaine d'application serait bien délimité. Elle devrait laisser la plupart des responsabilités pratiques d'actions préventives aux cantons ou à des organisations privées, tout en encourageant la collaboration régionale et intercantonale. 